\title{
EFFECTS OF CT INJECTOR ACCELERATION ELECTRODE CONFIGURATION ON TOKAMAK PENETRATION
}

\author{
J. YEE, P.M. BELLAN \\ California Institute of Technology, \\ Pasadena, California, \\ United States of America
}

ABSTRACT. Through compact toroid (CT) injection experiments on the TEXT-U tokamak (with $B_{\mathrm{T}} \simeq 10 \mathrm{kG}$ and $I_{\mathrm{P}} \simeq 100 \mathrm{kA}$ ), it has been shown that the acceleration electrode configuration, particularly in the vicinity of the toroidal field (TF) coils of the tokamak, has a strong effect on penetration performance. In initial experiments, premature stopping of CTs within the injector was seen at anomalously low TF strengths. Two modifications were found to greatly improve performance: (a) removal of a section of the inner electrode and (b) increased diameter of the 'drift tube' (which guides the CT into the tokamak after acceleration). It is proposed that the primary drag mechanism slowing CTs is toroidal flux trapping, which occurs when a CT displaces transverse TF trapped within the flux conserving walls of the acceleration electrodes (or drift tube). For a simple two dimensional (2-D) geometry, a magnetostatic analysis produces a CT kinetic energy requirement of $\frac{1}{2} \rho v^{2} \geq \alpha\left(B_{0}^{2} / 2 \mu_{0}\right)$, with $\alpha=2 /\left(1-a^{2} / R^{2}\right)$ a dimensionless number that is dependent on the CT radius $a$ normalized by the drift tube radius $R$. For a typical CT, this can greatly increase the required energies. A numerical analysis in 3-D confirms the analytical result for long CTs (with length $L$ such that $L / a \gtrsim 10$ ). In addition to flux trapping, the CT shape is also shown to affect the energy criterion. These findings indicate that a realistic assessment of the kinetic energy required for a CT to penetrate a particular tokamak TF must take into account the interaction of the magnetic field with the electrode walls of the injector.

\section{INTRODUCTION}

Acceleration of compact toroid (CT) spheromaks offers the potential of a viable refuelling technique for reactor grade tokamak plasmas $[1,2]$. Compact toroid spheromaks are nearly force-free axisymmetric plasma rings in which the toroidal and poloidal magnetic fields are of comparable magnitude and have the desirable properties of stability, robustness and long lifetimes, allowing acceleration to very high velocities [3].

According to current theories, one of the key requirements for the success of $\mathrm{CT}$ injection schemes is that the plasmoid must attain sufficient kinetic energy density in order to overcome the strong gradient of the tokamak toroidal field (TF) [1]. Owing to the short time-scales involved in the injection process and the high electrical conductivity of the plasma, the tokamak TF must be diamagnetically displaced by the travelling $\mathrm{CT}$. The CT encounters a retarding force per unit volume $\boldsymbol{F}_{\mathrm{CT}} / V=-\boldsymbol{\nabla}\left(B^{2} / 2 \mu_{0}\right)$ analogous to the buoyancy force an object experiences when submersed in a liquid, with magnetic pressure taking the place of hydrostatic pressure. Thus, the initial translational kinetic energy of the CT must be sufficiently high in order to penetrate a given mag- netic field. On the basis of this argument, the kinetic energy requirement is

$$
\frac{1}{2} \rho v^{2}>\frac{B_{\mathrm{T}}^{2}}{2 \mu_{0}}
$$

where $\rho$ is the average mass density of the $\mathrm{CT}, v$ is the velocity of the $\mathrm{CT}$ after acceleration and $B_{\mathrm{T}}$ is the tokamak TF strength. In physical terms, the initial kinetic energy density of the CT must exceed the magnetic energy density of the tokamak TF. We shall show later that this criterion is not always accurate and may significantly underestimate the actual kinetic energy requirement for successful penetration.

In addition to toroidal magnetic field displacement, other drag mechanisms have also been predicted. In Parks' model [2], CT motion through the tokamak plasma excites a wake of Alfvén waves that drains kinetic energy from the CT. The resulting drag force on the CT is $F_{\text {Parks }}=\frac{1}{2} B_{\mathrm{T}}^{2} a^{2}\left(v / v_{\mathrm{A}}\right)$, where $v_{\mathrm{A}}$ is the Alfvén speed and $a$ is the CT radius. Newcomb [4] included power radiated in the form of magnetoacoustic waves and arrived at an expression $F_{\text {Newcomb }}=2 \pi \rho a^{2} I v c_{\mathrm{s}}$, where $c_{\mathrm{s}}$ is the speed of sound and $I$ is a drag coefficient of order 1 . These additional sources of drag would further slow the CT, increasing the kinetic energy requirement. 
(a)

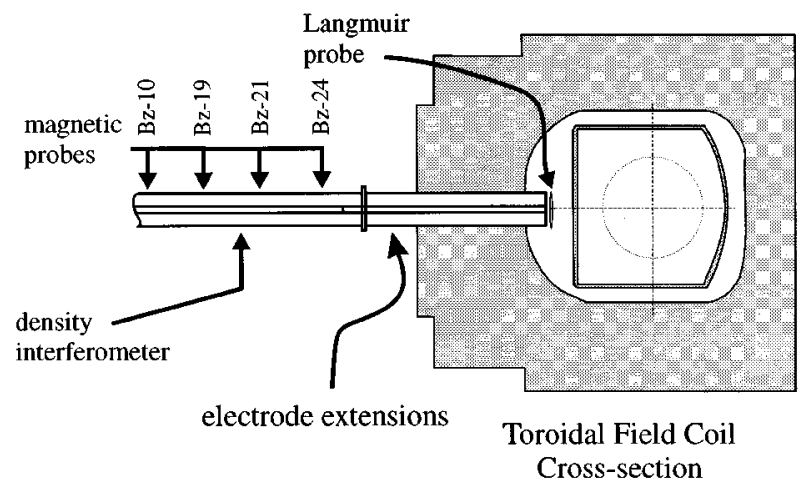

(b)

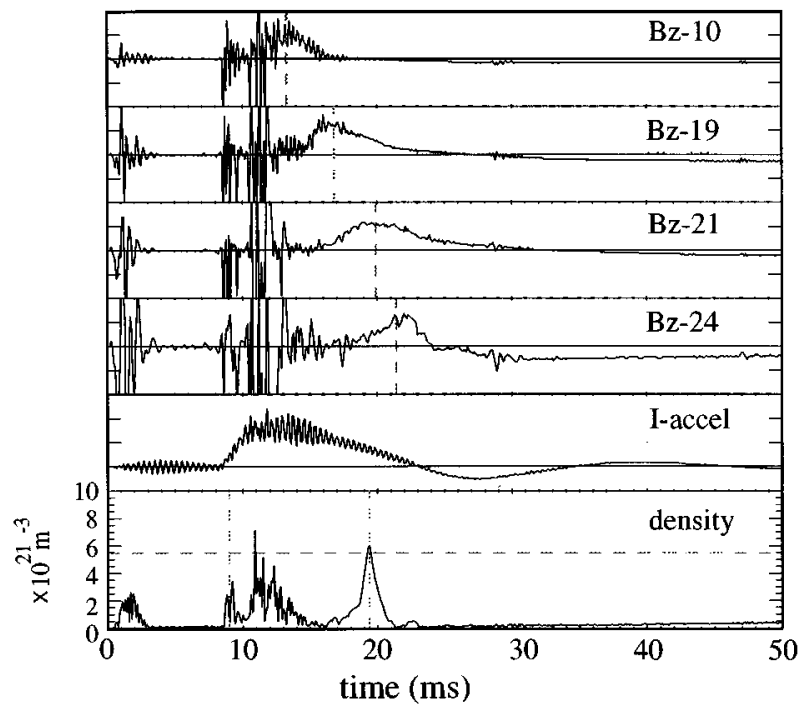

To date, there have been several experimental investigations of CT injection into tokamaks. Initial experimental demonstrations of injection of an unaccelerated CT into a small tokamak [5] have been followed by non-disruptive injection of CTs into the medium sized TdeV tokamak [6].

We present here results from CT injection experiments using the Caltech CT Injector on the University of Texas at Austin TEXT-U tokamak operating at TF strengths $B_{\mathrm{T}} \approx 10 \mathrm{kG}$. Various acceleration electrode configurations were examined, and it was discovered that electrode modifications had pronounced effects on penetration.

\section{EXPERIMENTAL SET-UP}

The Caltech CT injector [7] consists of a two stage coaxial electrode system, comprised of a formation section and an acceleration section. The outer elec-

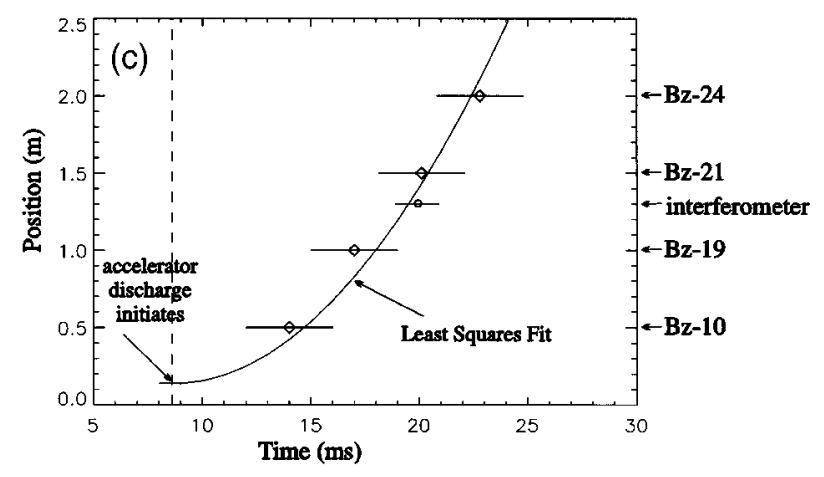

FIG. 1. (a) Cross-sectional view of the tokamak, including one TF coil and the CT injector electrodes. Both inner and outer electrode extensions are shown. The tokamak vessel and TF coil are drawn to scale, while the CT injector diagnostic positions are approximate. (b) Sample data from a CT shot. The peak gun current is $100 \mathrm{kA}$ and the peak density is $6 \times 10^{21} \mathrm{~m}^{-3}$. (c) Plot showing a velocity calculation. The data points denote the time of passage of a CT based on magnetic probe or interferometer measurements. The curve is the least squares fit of the five data points to a constant acceleration model. This results in a calculated velocity at the interferometer $(x=1.3 \mathrm{~m})$ of $3 \times 10^{5} \mathrm{~m} / \mathrm{s} \pm 30 \%$, corresponding to a kinetic energy density of about $4 \times 10^{5} \mathrm{~J} / \mathrm{m}^{3}$.

trode is copper spray coated with tungsten in the formation region, and becomes copper shrouded tantalum in the acceleration region. The inner electrode is a $6.4 \mathrm{~cm}$ diameter tantalum sheath surrounding a copper tube. The copper ensures that both electrodes are good magnetic flux conservers on the experimental time-scale, so that the decay of the CT magnetic helicity is minimized. The electrodes are baked at $200^{\circ} \mathrm{C}$ prior to operation of the injector in order to remove adsorbed impurities; this cleaning operation enhances CT performance.

The CT formation stage uses a magnetized Marshall gun configuration. Four high speed valves puff hydrogen or deuterium gas into the region between the inner and outer electrodes, and 8 to $10 \mathrm{kV}$ is then applied across this gap using an ignitron switched $120 \mu \mathrm{F}$ capacitor bank. The high voltage breaks down the gas and subsequently drives current through the resulting plasma, creating a toroidal magnetic field embedded ('frozen') in the plasma. If the formation 
current is sufficiently large, the intrinsic $\boldsymbol{J} \times \boldsymbol{B}$ force pushes the plasma through the externally applied poloidal magnetic field. Magnetic field lines then reconnect behind the resultant plasma ring, and the plasma relaxes to a near force-free compact torus (i.e. spheromak) configuration.

After the CT has been formed, another ignitron circuit discharges a second capacitor bank $(180 \mu \mathrm{F}$ at $\approx 11 \mathrm{kV}$ ), accelerating the $\mathrm{CT}$ through the $\approx 3 \mathrm{~m}$ acceleration section, again using the $\boldsymbol{J} \times \boldsymbol{B}$ forces, with the CT acting as the armature of a coaxial railgun. Four magnetic pick-up probes spaced at $0.5 \mathrm{~m}$ intervals along the outer electrode provide time of flight measurements, a helium-neon laser interferometer measures the density at a point late in the acceleration phase and a Langmuir probe measures the ion current of the plasma as it makes its exit from the injector (Fig. 1(a)). A sample shot is shown in Fig. 1(b).

The density interferometer is of a quadrature type [8], utilizing a linearly polarized scene beam and a circularly polarized reference beam to produce two separate output signals. This avoids calibration difficulties and eliminates data interpretation ambiguities due to fringe skipping. The time at which the passage of the $\mathrm{CT}$ is detected by each of the four magnetic probes is determined by numerically smoothing the voltage trace and directly finding the time at which the maximum value occurs. These times, along with the time of peak density, are least squares fits (LSFs) to a uniform acceleration model as shown in Fig. 1(c), using the data from Fig. 1(b). The LSF gives a velocity of $3 \times 10^{5} \mathrm{~m} / \mathrm{s}$ at the location of the interferometer. This value, when combined with the peak density value of $6 \times 10^{21} \mathrm{~m}^{-3}$, corresponds to a kinetic energy density of $4 \times 10^{5} \mathrm{~J} / \mathrm{m}^{3}$. On the basis of the calculated velocity, the FWHM of the density pulse corresponds to a CT axial length of about $0.5 \mathrm{~m}$.

Uncertainty in the value of the kinetic energy density arises mainly from measurement uncertainties in determining the final velocity of the $\mathrm{CT}$, which may be of the order of $30 \%$. This relatively high uncertainty must be kept in mind in the discussions below. We can use a simple energy argument to verify that this estimate of the kinetic energy density is, at the very least, reasonable. The total kinetic energy contained in the CT is $2 \mathrm{~kJ}$. Comparing this with the total gun and accelerator capacitor bank energies of $16 \mathrm{~kJ}$ gives a moderate energy efficiency of $12.5 \%$.

Figure 1(a) shows a cross-sectional view of the TEXT-U tokamak, including one TF coil with the CT injector acceleration electrodes superimposed. The

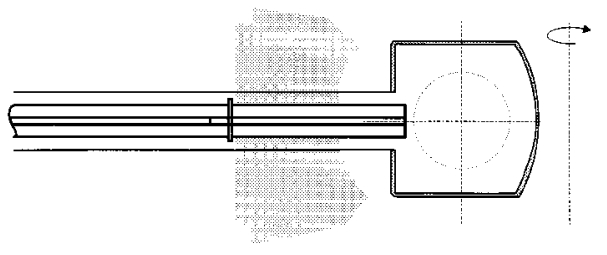

(a) Electrode Extensions On (Case I)

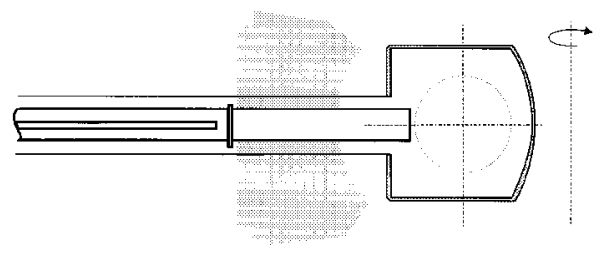

(b) Central Electrode Extension Removed (Case II)

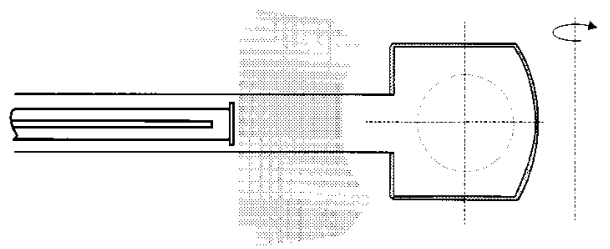

(c) Both Electrode Extensions Removed (Case III)

FIG. 2. Diagram of the three electrode configurations used during the CT injector experiments: (a) electrode extensions both attached (case I), (b) inner electrode extension removed (case II), (c) both electrode extensions removed (case III). In the latter case, CTs are confined by the vacuum vessel walls once they have left the injector.

$\mathrm{CT}$ injector was connected to the tokamak vacuum chamber with vacuum bellows, and the entire injector assembly was mounted on rails, allowing adjustment of the longitudinal positioning of the injector relative to the tokamak plasma. TEXT-U is a medium sized tokamak, with major radius $1.05 \mathrm{~m}$ and minor radius $27 \mathrm{~cm}$. The TEXT-U diagnostics include multichord density interferometers, soft $\mathrm{X}$ ray detectors and Mirnov MHD coils. Compact toroid injection experiments were conducted in a limiter configuration, with $\approx 10 \mathrm{kG}$ toroidal fields and $\approx 100 \mathrm{kA}$ plasma currents. Some experiments were performed at lower TFs by firing the injector during the decaying portion of the TF, after the tokamak discharge.

\section{RESULTS}

Compact toroid injector experiments were conducted with three different configurations of the acceleration electrodes, as shown in Fig. 2, and it was found that the choice of configuration 

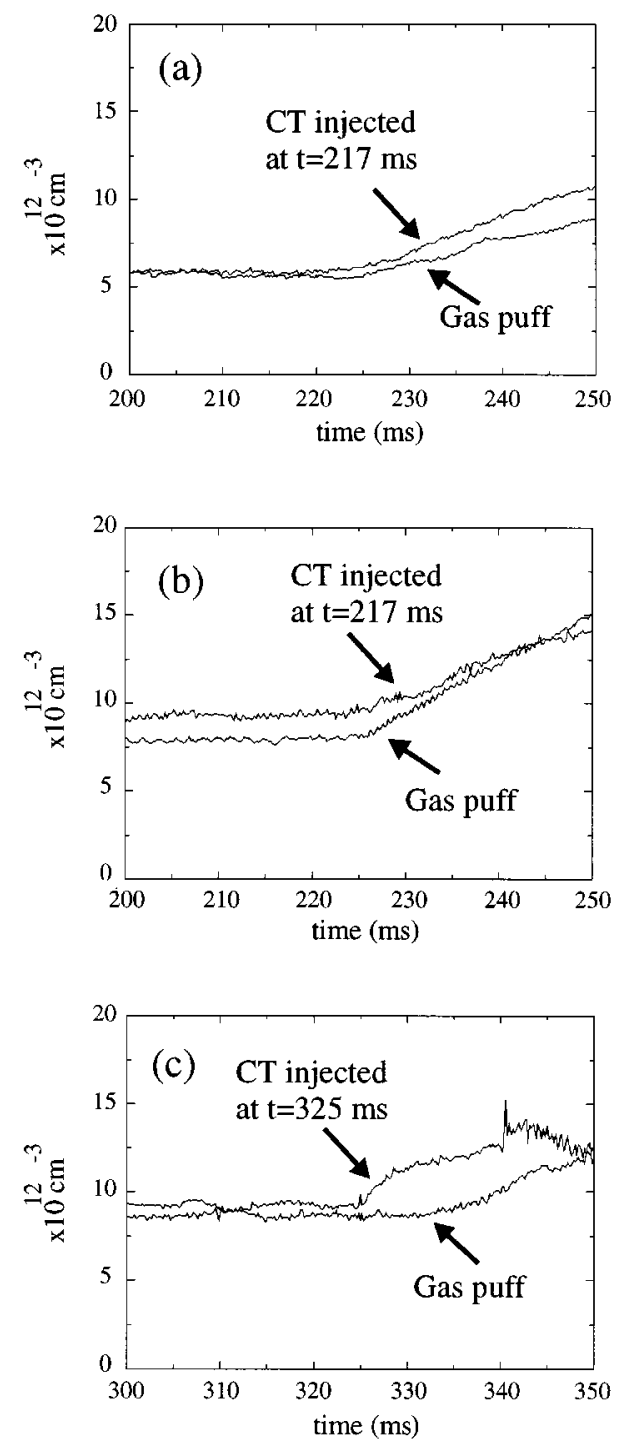

FIG. 3. Tokamak density interferometer traces (central chord) for each electrode configuration (see the corresponding parts of Fig. 2). In all cases, the tokamak TF is $10 \mathrm{kG}$ and the plasma current is $100 \mathrm{kA}$. In each plot, the tokamak density upon CT injection is compared with the tokamak density following puffing of the CT injector gas valves used in CT formation.

profoundly affected the CT penetration into the tokamak. The key feature distinguishing the various electrode geometries is the position of the ends of the electrodes relative to the TF coils of TEXT-U. The initial configuration (case I, Fig. 2(a)) included extension sections to both the central and the outer electrodes, to provide additional acceleration, compression and confinement of the $\mathrm{CT}$ into a region just outside the outermost flux surface of the tokamak plasma. The outer electrode extension is tapered from $12.7 \mathrm{~cm}$ diameter to $11.3 \mathrm{~cm}$ over its $0.74 \mathrm{~m}$ length. In the second configuration (case II), shown in Fig. 2(b), the central electrode was shortened by removing the extension section. Figure 2(c) shows the final configuration (case III), in which the outer electrode extension was also removed, terminating the acceleration electrode assembly outside the extent the TF coils.

\subsection{Electrode extensions on (case I)}

Initial experiments, with both central and outer electrode extensions attached, exhibited surprisingly poor penetration by the CT. Figure 3(a) shows tokamak interferometry data for a discharge with $B_{\mathrm{T}}=$ $10 \mathrm{kG}$, with CT injection occurring at $t=217 \mathrm{~ms}$. The lower trace on this same plot shows the tokamak density for a shot in which the $\mathrm{CT}$ injector gas valves are fired, but the formation and acceleration capacitor banks are not discharged. The similarity of the two cases indicates that the density increase in the tokamak starting at $t=217 \mathrm{~ms}$ is due to diffusion of neutrals, and not CT injection. Magnetic probes and interferometry data on the $\mathrm{CT}$ injector indicate an estimated CT kinetic energy density of $4 \times 10^{5} \mathrm{~J} / \mathrm{m}^{3}$, so that the standard minimum energy criterion given by Eq. (1) is satisfied.

Further direct evidence of the lack of CT penetration was provided by measurements using a Langmuir probe mounted at the end of the acceleration (outer) electrode, positioned to detect the presence of plasma leaving the end of the injector. Lack of a signal from this diagnostic indicates that the $\mathrm{CT}$ has slowed to a stop within the final section of the injector. Figure 4(a) shows the peak signal from this probe as a function of tokamak TF strength. Each data point represents a $\mathrm{CT}$ injection shot in which the estimated kinetic energy density is $3 \times 10^{5}$ to $4 \times 10^{5} \mathrm{~J} / \mathrm{m}^{3}$, corresponding to a maximum $\mathrm{TF}$ of $10 \mathrm{kG}$. ( $\mathrm{TF}$ values of less than $10 \mathrm{kG}$ were obtained by firing the $\mathrm{CT}$ injector during the decay of the $\mathrm{TF}$, after the tokamak discharge.) The amount of plasma that leaves the injector is obviously highly dependent on the TF, with a threshold value of $2 \mathrm{kG}$, much lower than the predicted $10 \mathrm{kG}$.

\subsection{Central electrode extension removed (case II)}

In the second electrode configuration, the central electrode extension was removed, shortening this electrode. This modification reduces the length of the acceleration region, and the final section of the outer electrode is converted into a 'drift tube', where 


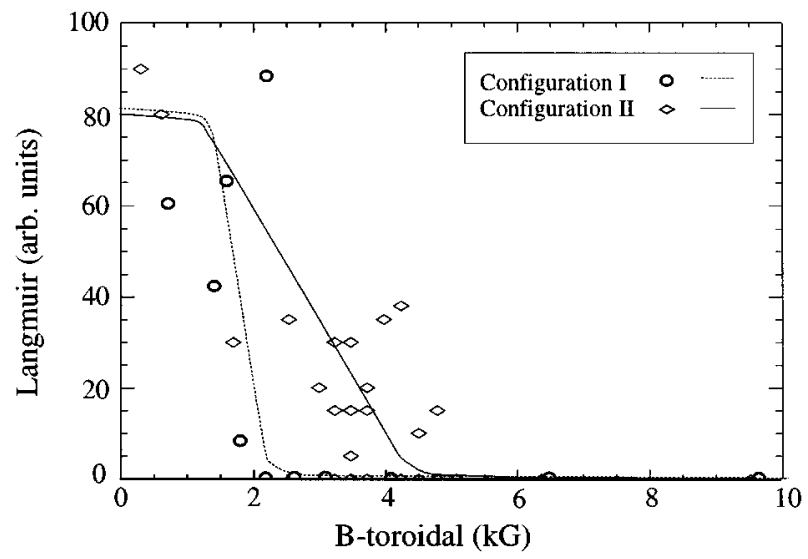

FIG. 4. Langmuir probe measurements for the first two electrode configurations, I and II. Each point represents the maximum CT Langmuir signal detected at the end of the injector, for a particular tokamak TF strength.

the CT must travel into the tokamak by virtue of its initial momentum. Despite the reduction in the acceleration stage, injection performance was notably improved in this configuration, as compared with that in case I. Langmuir probe measurements and tokamak interferometry data are shown in Figs 3(b) and 4(b), respectively. As shown in Fig. 4(b), the TF magnitude above which no plasma can be detected leaving the injector increased to approximately $4.5 \mathrm{kG}$. However, at the tokamak's operating TF strength of $10 \mathrm{kG}$, there is still no plasma detected leaving the injector. Figure $3(\mathrm{~b})$ shows the density trace of a $10 \mathrm{kG}$ tokamak discharge, upon $\mathrm{CT}$ injection at $t=217 \mathrm{~ms}$, with an estimated CT kinetic energy density of $4 \times 10^{5} \mathrm{~J} / \mathrm{m}^{3}$. Comparing this with the reference gas puffing injection, the data indicate that the bulk of the CT mass has not penetrated into the tokamak, and the density rise after the $\mathrm{CT}$ injection time is again due to neutral particle diffusion.

\subsection{Both electrode extensions removed (case III)}

In the third configuration, the outer electrode extension was also removed, resulting in an effective drift tube consisting of the larger stainless steel vacuum bellows cylinder $(\approx 20 \mathrm{~cm}$ inner diameter $)$. Unfortunately, accessibility problems prevented a Langmuir probe from being mounted as in the previous configurations. Sample tokamak data are shown in Fig. 5, for a $10 \mathrm{kG}$ tokamak discharge, with CT injection occurring at $t=325 \mathrm{~ms}$, and an estimated CT kinetic energy density of $4 \times 10^{5} \mathrm{~J} / \mathrm{m}^{3}$. Note the sharp (several millisecond time-scale) rise in density upon CT injection, which is higher than can be

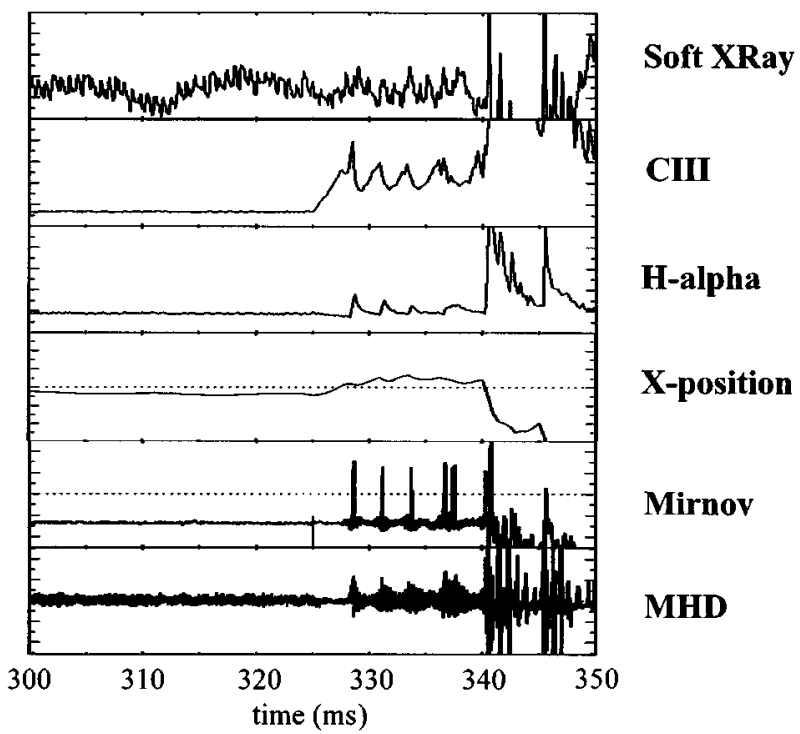

FIG. 5. Tokamak diagnostics for configuration III with tokamak parameters: $B_{\mathrm{T}}=10 \mathrm{kG}$ and $I_{\mathrm{p}}=100 \mathrm{kA}$. Compact toroid injection occurs at $t=325 \mathrm{~ms}$.

accounted for by simple neutral gas diffusion, indicating penetration of the $\mathrm{CT}$ to a point nearer to the outermost flux surface of the tokamak. The X position trace shows the horizontal location of the imaginary plasma current 'filament' in a particular poloidal cross-section, measured using magnetic probes (used for active feedback stabilization of the discharge). This latter plot indicates a sharp (several millisecond) motion of the plasma column upon injection. Soft X ray, $\mathrm{H}$ alpha, Mirnov and MHD probe measurements show a great deal of MHD activity after CT injection, with a particularly strong component with $3 \mathrm{~ms}$ periodicity, resulting in a minor tokamak disruption at $t=341 \mathrm{~ms}$. These diagnostics clearly demonstrate that $\mathrm{CT}$ injection dramatically affects the tokamak discharge. In contrast, gas puff reference shots exhibit none of this behaviour.

A few injection shots were attempted at $\mathrm{TF}$ strengths of $7 \mathrm{kG}$ (below the normal operating regime of TEXT-U), with the expectation of increased penetration. In these cases, the results were qualitatively similar to those described for $10 \mathrm{kG}$.

\section{TOROIDAL FLUX CONSERVATION (TRAPPING)}

The results described above suggest that interaction between a $\mathrm{CT}$ and the $\mathrm{TF}$ of the tokamak results in slowing down of the $\mathrm{CT}$ to an extent far greater than can be explained using the standard energy 
criterion (Eq. (1)). Since the drag occurs in the final stage of the injector, where no stationary background plasma is present, the drag mechanisms proposed by Parks and Newcomb, which involve energy loss via emission of Alfvén or acoustic waves, are not relevant. Only the force due to the gradient of the toroidal field $\nabla\left(B^{2} / 2 \mu_{0}\right)$ is applicable in this region. Clearly, this force does not adequately account for CT slowing, since CTs with the kinetic energies that we have estimated to be sufficient to penetrate $10 \mathrm{kG}$ become stalled within the injector and do not emerge at TF strengths as low as $2 \mathrm{kG}$.

In attempting to interpret these anomalous results, let us first consider the possibility that the kinetic energy of the accelerated CT has simply been overestimated. Substantial measurement uncertainties in the final velocity, as discussed earlier, as well as possible slowing of the $\mathrm{CT}$ due to electrode surface effects could potentially result in significantly reduced final energies. However, it seems unlikely that these factors can account for the $80 \%$ reduction in velocity (and correspondingly greater reduction in energy) that would be required. We will therefore restrict our attention to stopping mechanisms related to the tokamak TF.

We hypothesize that the anomalously poor penetration is attributable to TF being trapped within the flux conserving walls of the coaxial electrodes. Owing to the finite size of the coils that generate the TF, the tokamak TF drops off gradually outside the tokamak. In the case of TEXT-U, the field decays from $77 \%$ of the nominal value at the tokamak plasma edge down to $25 \%$ a distance $40 \mathrm{~cm}$ away from the edge. (The nominal value of the TF is measured at the centre of the tokamak discharge.) This residual $\mathrm{TF}$ will then transversely permeate the $\mathrm{CT}$ injector electrodes, which are flux conserving on the relevant microsecond time-scales. Thus, the field lines are effectively 'frozen in' or trapped within the electrode walls, impeding the progress of the CT plasma, which is also flux conserving and must displace these field lines as it travels into the high field region.

We first examine the effect of the TF on the electrode geometries II and III, in which magnetic flux is trapped within the outer electrode (or drift tube). In this case, shown in Fig. 6(a), the energy required for the CT plasma to displace the TF lines can be greatly increased, as compared with the case in which no electrode is present, since any field that is displaced must be compressed against the walls. The TF magnetic flux is effectively trapped inside the drift tube, resulting in an increase in the magnetic field (a)

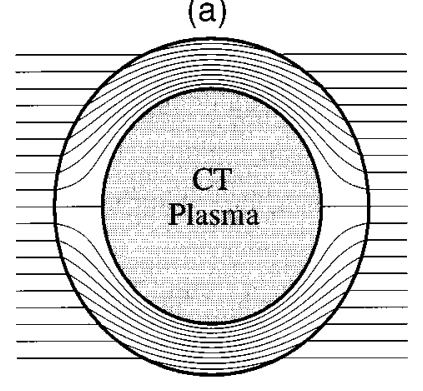

(b)

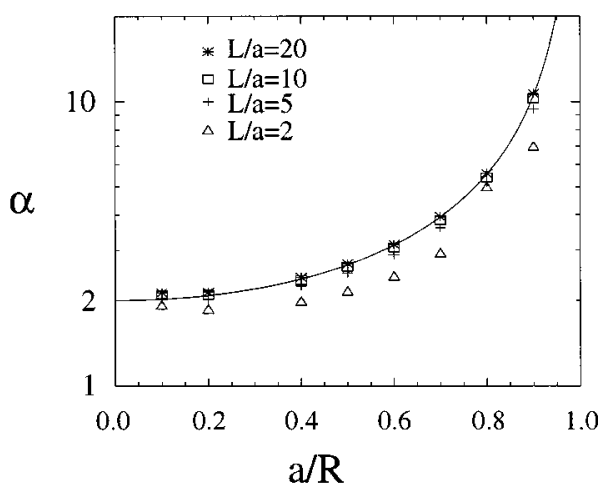

(c)

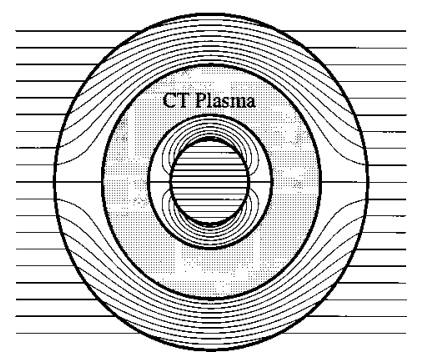

FIG. 6. Toroidal flux trapping. (a) Cross-sectional view of magnetic field topology for the case of a circular CT plasma within a circular drift tube. (b) Magnetic energy enhancement factor $\alpha$ as a function of CT radius $a$ normalized by drift tube radius $R$. The solid curve shows the 2 -D analytical expression. The data points were numerically calculated for various values of $L / a$. (c) Crosssectional view of the magnetic field for the case of an annular CT plasma, with both inner and outer electrodes present.

strength in the vicinity of the CT. We first use a simple two dimensional (2-D) magnetostatic analysis to calculate the change in magnetic field energy resulting from the flux displacement caused by the plasmoid and then use numerical methods to investigate the full 3-D case.

The axial extent of our CT greatly exceeds its radius, so that a $2-\mathrm{D}$ analysis is appropriate. We assume that the (uniform) TF field has had sufficient 
time to fully penetrate the walls of the drift tube, and is 'frozen in' on the CT time-scale. Furthermore, for simplicity, we will assume an axisymmetric, incompressible CT. The cylindrical CT drifts from infinity (a field-free region) into this high $\mathrm{TF}$ region, and must fully exclude these magnetic field lines, since the CT is itself a flux conserver.

We thus consider a magnetically impermeable body of radius $a$ within a larger ring of inner radius $R$, with a uniform magnetic field of strength $B_{0}$ frozen inside it. In a 2-D polar co-ordinate system, the general (current-free) magnetic field is $\boldsymbol{B}=\boldsymbol{\nabla} \Psi$, where the potential function $\Psi$ is

$\Psi(r, \theta)=\sum_{m}\left(c_{1 m} r^{m}+c_{2 m} r^{-m}\right) \sin m \theta$.

Applying the boundary conditions, $\left[B_{r}\right]_{r=R}=$ $B_{0} \sin \theta$ and $\left[B_{r}\right]_{r=a}=0$, gives

$\Psi=B_{0} r\left(1+\frac{a^{2}}{r^{2}}\right) \frac{R^{2}}{R^{2}-a^{2}} \sin \theta$.

The total magnetic field energy within a cylindrical control volume $V$ is given by

$W=\int_{V} \frac{1}{2 \mu_{0}}|\nabla \Psi|^{2} \mathrm{~d}^{3} x$.

Before the CT enters the drift tube, the control volume is permeated by a uniform field of magnitude $B_{0}$, so that the magnetic field energy is simply $W_{0}=\left(B_{0}^{2} / 2 \mu_{0}\right) V$. Thus, the net change in magnetic field energy resulting from the addition of the flux excluding body in a section of length $L$ is

$\Delta W=W-W_{0}=\frac{2 R^{2}}{R^{2}-a^{2}} \frac{B_{0}^{2}}{2 \mu_{0}}\left(\pi a^{2} L\right)$.

If we now identify $V_{\mathrm{CT}}=\pi a^{2} L$ as the volume of the $\mathrm{CT}$, and apply conservation of energy, $\Delta W+$ $\Delta K=0$, the change in kinetic energy of a $\mathrm{CT}$ as it enters a flux conserving drift tube is then

$\Delta K=-\frac{2 R^{2}}{R^{2}-a^{2}}\left[\left(\frac{B_{0}^{2}}{2 \mu_{0}}\right)\left(V_{\mathrm{CT}}\right)\right]$.

This requires the $\mathrm{CT}$ to have a minimum initial kinetic energy $K_{0} \geq \alpha\left(B_{0}^{2} / 2 \mu_{0}\right) V_{\mathrm{CT}}$ in order to displace the magnetic field trapped inside the drift tube, where

$\alpha \equiv \frac{2 R^{2}}{R^{2}-a^{2}}$.

In order to arrive at a form analogous to the standard criterion, the $\mathrm{CT}$ total kinetic energy must be rewritten in terms of its average kinetic energy density,
$K_{0}=\frac{1}{2} \rho v^{2} V_{\mathrm{CT}}$, where $\rho$ and $v$ are the CT average density and initial velocity, respectively. The criterion for the required CT kinetic energy density becomes

$\frac{1}{2} \rho v^{2} \geq \alpha \frac{B_{0}^{2}}{2 \mu_{0}}$.

This modified energy criterion differs from the standard expression only in the multiplicative factor $\alpha$, which can be interpreted as a measure of the enhancement of the retarding force on the CT as it displaces field lines in a flux trapped region. Note that $\alpha$ is proportional to the ratio between the crosssectional area of the drift tube $\pi R^{2}$ and the area between the CT and the drift tube $\pi\left(R^{2}-a^{2}\right)$. In addition, $\alpha$ depends strongly on the ratio between the radius $a$ of the $\mathrm{CT}$ plasma and the drift tube inner radius $R$ (cf. Fig. 6(b)); this ratio is determined by magnetic and thermal pressure balance, as well as the dynamics of the deceleration process. Furthermore, $\alpha$ can be quite large, and even in the most energetically favourable case, which is the limit of an infinitely thin $\mathrm{CT}$, approaches a minimum value of 2 . This implies that deceleration of the CT may frequently be dominated by this mechanism, so that the critical CT kinetic energy required for penetration is determined by Eq. (8), rather than the commonly used Eq. (1).

One may, at first, be surprised by the limiting value of $\alpha_{\min }=2$ derived for the case of a drift tube with infinitely large diameter. In this situation, the walls of the drift tube have no effect upon the magnetic field around the CT, and thus flux trapping cannot be an issue. Instead, the value of 2 stems entirely from the boundary conditions on the surface of the CT (and hence on the shape of the CT). These surface boundary effects on the magnetic field were incorrectly neglected in the earlier argument (leading to Eq. (1)). Unlike the case of an object submersed in a liquid, in which the fluid pressure simply increases linearly with depth, the magnetic field (and thus the magnetic pressure) around a flux excluding $\mathrm{CT}$ is influenced by the shape of the object. As a result, the deceleration force that the $\mathrm{CT}$ experiences is inherently dependent upon its shape and this is quantitatively reflected in the value of $\alpha_{\min }$. Note that this issue is entirely separate from the flux trapping effect resulting from the confining influence of the drift tube.

The value of $\alpha_{\min }$ appropriate for any particular CT shape can be calculated using a magnetostatic analysis. In addition to the value of 2 calculated above for a long cylindrical $\mathrm{CT}$ that is oriented perpendicular to the magnetic field, it is illuminat- 
ing to examine two other cases that can be solved analytically. Straightforward calculations show that $\alpha_{\min }=3 / 2$ would be applicable for a spherical CT (i.e. the 'classical' spheromak) and $\alpha_{\text {min }}=1$ would be applicable for a long thin cylinder oriented parallel to the magnetic field (cf. Appendix). These latter results reflect the fact that there is an energetic preference for thin objects with a small profile perpendicular to the magnetic field, because this results in the least distortion of field lines; the minimum value of $\alpha_{\min }=1$ can be regarded as an absolute lower bound for $\alpha$. A general consequence of this observation is that any diamagnetic object in a magnetic field will tend towards a shape or orientation that reduces its transverse dimensions (i.e. cross-section).

In order to ascertain the effect of including finite CT axial length in this model, a numerical calculation was also performed. In the computational model, the CT is a cylinder of radius $a$ and length $2 L$ located within a larger cylinder of radius $R$ and length $5 L$. (The length of the outer cylinder was chosen so that it did not significantly affect the result.) By assuming that $\Psi \propto \sin \theta$, as is required by the boundary conditions, the azimuthal co-ordinate is eliminated, transforming the problem into a 2-D one (in $r$ and $z$ ). The region is discretized into 400 intervals in the radial direction and 100 intervals in the axial direction. The finite element differential equation $\nabla^{2} \Psi=0$, along with the appropriate boundary conditions, was then solved using a successive over-relaxation (SOR) algorithm, performing 10000 iterations. The total magnetic energy in the volume could then be calculated numerically, and the kinetic energy criterion could be evaluated in a manner identical to that used in the analytical model above. In Fig. 6(b), the calculated values for $\alpha$ are shown for several CT aspect ratios $(L / a)$. The numerical values closely approach those predicted by the previous 2-D analytical model for aspect ratios of about 10 and higher. This implies that the analytical model should be a good approximation for the present case, where the measured aspect ratio is $L / a \approx 5$. For smaller aspect ratios, the analytical model may modestly overestimate the required $\mathrm{CT}$ kinetic energy.

A similar calculation can also be performed for configuration I, in which a transverse DC magnetic field is trapped within both the central and outer electrodes. The resulting magnetic field structure is shown in Fig. 6(c), with an annular CT cross-section. In this case, the amplification factor $\alpha$ depends on two additional quantities: the inner radius of the CT and the radius of the central electrode. The essential physics remains the same, and the required kinetic energy is increased by an amount that is sensitive to the dimensions of the $\mathrm{CT}$. The notable difference, however, lies in the decreased cross-sectional area that the CT is permitted to occupy. As discussed above, increased drag is caused when the magnetic field strength is magnified by TF trapping within the limited area between the $\mathrm{CT}$ and the electrode walls. Thus, if we assume an incompressible CT, removal of the central electrode in this region would increase the space available for the magnetic field and reduce the effects of TF trapping.

The influence on injector performance of toroidal $\mathrm{TF}$, with and without the central electrode, can be quantified by comparing the results obtained with the electrode configurations I and II. In the latter geometry, removal of the central electrode converts the region into a simple drift tube, with a $30 \%$ increase in cross-sectional area. As described above, the Langmuir probe results indicate an increase of the magnetic field threshold value from 2 to $4.5 \mathrm{kG}$, as compared with configuration I. Thus, the presence of the central electrode within the TF region does appear to have a detrimental effect on performance, with an approximately fivefold reduction in CT energy in our experiment.

Our results are also consistent with this analysis of flux trapping within a drift tube. Both the configurations II and III involve final drift tube sections, with differing diameters. In the latter case, the $20 \mathrm{~cm}$ inner diameter stainless steel vacuum bellows comprises the drift tube. (Although stainless steel is a relatively poor electrical conductor, it is still a magnetic flux conserver for the time-scales of interest.) The improved performance of geometry III is due to the increased drift tube diameter $R$, reducing the critical ratio $a / R$, and hence reducing the decelerating effect of flux trapping.

\section{DISCUSSION}

Clearly, in the final configuration III, the increased effects on the tokamak diagnostics such as density and radiation measurements are indicative of greatly improved CT penetration through the drift tube, as compared with those of the earlier configurations. Despite this improvement, an analysis of diagnostic data indicates that CTs have still not been successfully injected into the tokamak at the operational $\mathrm{TF}$ strength of $10 \mathrm{kG}$. Injection of a CT occurs on a time-scale of several microseconds, followed by a 
period of disintegration, which should require less than $100 \mathrm{~ms}$, applying either of the two disassembly mechanisms proposed by Parks [2]. Rapid $(<0.5 \mathrm{~ms})$ density increase accompanied by a similarly fast soft $\mathrm{X}$ ray drop, upon $\mathrm{CT}$ injection, was reported by Raman et al., in an experiment with similar CT and tokamak parameters [6]. The several millisecond risetime in tokamak density measured in this experiment is much longer than can be accounted for by $\mathrm{CT}$ decay, and suggests that CTs have not fully penetrated into the tokamak plasma.

The reasons for the lack of CT penetration are unclear, since the details of CT behaviour and dynamics within the drift tube are not understood. We present here two possible scenarios that would lead to failure of the CTs to penetrate. First, the CTs may stop towards the end of the drift tube, in spite of our electrode modifications. For example, stopping may occur as a result of the $\mathrm{TF}$ trapping mechanism proposed above, combined with an expansion of the CTs within the drift tube (and a corresponding decrease in kinetic energy density). The CTs subsequently decay and recombine, resulting in a high density of particles near the edge of the tokamak discharge, which then feeds the tokamak density through particle diffusion. Another possible stopping mechanism is contact with the drift tube walls, which were not baked to improve vacuum cleanliness, resulting in rapid decay of the CTs and deposition of their fuel at the tokamak edge. During earlier development of the $\mathrm{CT}$ injector, electrode surface impurities were linked to dramatic degradation in performance [9].

A second possibility that is consistent with the data is that the CTs arrive at the tokamak plasma edge, but are subsequently stopped upon interaction with the plasma. One would expect small cohesive plasmoid bodies to be able to penetrate the tokamak discharge, but as they travel within the drift tube, the CTs may expand both horizontally or vertically, and may be unable to maintain their structural integrity. Large and/or ill formed CTs that strike the tokamak plasma would probably be unable to penetrate to the interior, owing to the gross displacement and rearrangement of the TF tubes that would be required. In fact, this agrees rather well with the observed MHD behaviour of the tokamak upon injection. In particular, we have noted a shift in the tokamak plasma column beginning immediately after CT injection, which may be ascribed to the impulsive transfer of momentum from the CTs. This shock impulse generates MHD modes that quickly grow unstable and often lead to disruption. The sharp pulses in carbon impurity may be produced by motion of the plasma column resulting in contact with the limiter. This pattern of effects was observed both in $10 \mathrm{kG}$ discharges and in several $7 \mathrm{kG}$ discharges.

At this point, it is interesting to compare the present results with those reported by Raman on $\mathrm{TdeV}$. In the latter experiment, the electrode configuration most closely resembles our configuration III, with the end of the injector extending just slightly into the extent of the toroidal magnetic field [10]. That the TdeV results differ so strikingly from those reported here is most likely due to two factors. First, owing to a thinner, close fitting TF coil on the TdeV tokamak, the CTs need to travel only $17 \mathrm{~cm}$ within a drift tube in order to reach the entry port of the tokamak, as compared with $70 \mathrm{~cm}$ for the present experiment on the TEXT-U tokamak. The much shorter drift region reduces the effects of flux trapping and avoids expansion of the CTs. Second, the CT kinetic energies reported by Raman were based on average velocities, taken using time of flight measurements between two magnetic probes during the acceleration phase. Thus, the final velocities may have been significantly higher than reported, with correspondingly higher kinetic energies. Higher CT energies are also consistent with the much larger acceleration stage power supplies used in the latter experiment.

In light of the present results, we can make a few comments about the performance of CT injection systems. Clearly, close attention must be paid to the shape of the acceleration electrodes in the TF region. Focusing (or compression) of the CTs through tapering of the electrodes should ideally occur just outside the influence of the TF coils, followed by a larger diameter drift tube section that directs the CTs into the tokamak. However, care must be taken in minimizing the length of the drift tube region, and in avoiding excessive drift tube diameter, to provide some confinement of the CTs so that expansion does not occur to an unacceptable degree. The length and maximum diameter of the drift region will, in many practical cases, be constrained by the dimensions of the TF coil system and the entry port of the tokamak.

\section{Appendix}

In the above analysis of a cylindrical CT displacing magnetic field, it was shown that the retarding force is enhanced by a factor $\alpha$, which approaches a minimum value of 2 in the limit of infinitely distant confining walls, so that no flux trapping occurs. In this section, 
we will analyse $\alpha_{\min }$ for two other CT geometries. In each case, the flux excluding CT is first placed within an enclosure of finite dimensions with flux conserving walls. The magnetic field energy is then calculated to determine $\alpha$, and finally the size of the enclosure is allowed to grow to infinity.

\section{A1. Spherical CT}

We first consider the case of a spherical CT, using a spherical co-ordinate system in which the azimuthal axis is aligned with the direction of the uniform external magnetic field $B_{0}$. The $\mathrm{CT}$ is represented by a sphere of radius $a$ within a larger sphere of inner radius $R$, with magnetic field trapped within its walls. The configuration is similar to that shown in Fig. 6(a), and we will use the same approach as in the earlier analysis.

The general azimuthally symmetric (current-free) magnetic field is $\boldsymbol{B}=\boldsymbol{\nabla} \Psi$, where

$\Psi=\sum_{m}\left(c_{1 m} r^{m}+c_{2 m} r^{-(m+1)}\right) \cos m \theta$.

Applying the boundary conditions $\left[B_{r}\right]_{r=R}=$ $B_{0} \cos \theta$ and $\left[B_{r}\right]_{r=a}=0$ gives

$\Psi=B_{0} a \frac{R^{3}}{R^{3}-a^{3}}\left(\frac{r}{a}+\frac{a^{2}}{2 r^{2}}\right) \sin \theta$.

The total magnetic field energy within the volume between the two concentric spheres is given by

$W=2 \pi \int_{a}^{R} \mathrm{~d} r r^{2} \int_{0}^{\pi} \mathrm{d} \theta \sin \theta\left(\frac{1}{2 \mu_{0}}(\nabla \Psi)^{2}\right)$.

Before the CT enters the region and perturbs the field, the sphere of radius $R$ is permeated by a uniform field of magnitude $B_{0}$, so the magnetic field energy is simply $W_{0}=\left(B_{0}^{2} / 2 \mu_{0}\right)\left(4 \pi R^{3} / 3\right)$. Thus, the net change in magnetic field energy resulting from the addition of a flux excluding body in a section of length $L$ is

$\Delta W=W-W_{0}=\frac{3}{2} \frac{R^{3}}{R^{3}-a^{3}} \frac{B_{0}^{2}}{2 \mu_{0}}\left(\frac{4}{3} \pi a^{3}\right)$.

The volume of the CT is $V_{\mathrm{CT}}=4 \pi a^{3} / 3$, and by inspection $\alpha=(3 / 2) R^{3} /\left(R^{3}-a^{3}\right)$, so that the desired limit is $\alpha_{\min }=3 / 2$.

\section{A2. Cylindrical CT aligned with magnetic field}

The second case we consider is that of a long thin cylinder oriented parallel to the magnetic field. Suppose that the $\mathrm{CT}$ is represented by a cylinder of radius $a$ and length $L$, and is located within a larger cylinder of radius $R$ and length $L^{\prime}$. The appropriate boundary conditions are then simply that $B_{\perp}=0$ for all surfaces except for the ends $\left(z= \pm L^{\prime} / 2\right)$ of the larger cylinder, where $B_{\perp}= \pm B_{0}$. By integrating over a cylindrical volume, $\boldsymbol{\nabla} \cdot \boldsymbol{B}=0$ leads immediately to the condition that the magnetic flux $\Phi$ through any cross-section of the outer cylinder must be a constant. Using this result at one end $\left(z=L^{\prime} / 2\right)$ and at the centre $(z=0)$ gives

$B_{0} \pi R^{2}=\left.\int_{a}^{R} 2 \pi r B_{z}\right|_{z=0} \mathrm{~d} r$.

Let us now consider only the region defined by $-L / 2 \leq z \leq L / 2$ around the inner cylinder. In the limit of large $L$ (and $L^{\prime}$ ), edge effects can be ignored, and the magnetic field is spatially uniform and purely axial in this region. Thus, $\boldsymbol{B}=\left.B_{z}\right|_{z=0} \hat{\boldsymbol{z}}$ can be obtained directly from Eq. (13), and the total magnetic field energy is simply

$$
\begin{aligned}
W & =\frac{B^{2}}{2 \mu_{0}} \pi\left(R^{2}-a^{2}\right) L \\
& =\left(\frac{B_{0} R^{2}}{R^{2}-a^{2}}\right)^{2}\left(\frac{1}{2 \mu_{0}}\right) \pi\left(R^{2}-a^{2}\right) L \\
& =\left(\frac{B_{0}^{2}}{2 \mu_{0}}\right) \pi \frac{R^{4}}{R^{2}-a^{2}} L .
\end{aligned}
$$

In this case, the initial energy is $W_{0}=$ $\left(B_{0}^{2} / 2 \mu_{0}\right)\left(\pi R^{2} L\right)$, and the net change in energy is then

$\Delta W=W-W_{0}=\frac{R^{2}}{R^{2}-a^{2}} \frac{B_{0}^{2}}{2 \mu_{0}}\left(\pi a^{2} L\right)$.

Thus, $\alpha=R^{2} /\left(R^{2}-a^{2}\right)$ and the corresponding limit is $\alpha_{\min }=1$.

\section{ACKNOWLEDGEMENTS}

It is our pleasure to acknowledge the technical assistance of F. Cosso and L. Begay, and the considerable previous work of P. Loewenhardt and M. Brown in the development of the injector. We are indebted to R. Raman for sharing his knowledge and expertise with us. We would also like to thank W. Rowan and the entire TEXT-U team, whose co-operation and support made this work possible. We are also grateful to S. Sanders for helpful discussions.

This work was performed under USDOE Grant No. DE-FG03-86ER53232. 


\section{REFERENCES}

[1] PERKINS, L.J., HO, S.K., HAMMER, J.H., Nucl. Fusion 28 (1988) 1365.

[2] PARKS, P.B., Phys. Rev. Lett. 61 (1988) 1364.

[3] HAMMER, J.H., et al., Phys. Rev. Lett. 61 (1988) 2843.

[4] NEWCOMB, W.A., Phys. Fluids B 3 (1991) 1818.

[5] BROWN, M.R., BELlAN, P.M., Phys. Rev. Lett. 64 (1990) 2144.

[6] RAMAN, R., et al., Phys. Rev. Lett. 73 (1994) 3101.

[7] LOEWENHARDT, P.K., BROWN, M.R., YEE, J., BELLAN, P.M., Rev. Sci. Instrum. 66 (1995) 1050.

[8] BUChenaueR, C.J., JACOBSON, A.R., Rev. Sci. Instrum. 48 (1977) 769.
[9] BROWN, M.R., LOEWENHARDT, P.K., BELLAN, P.M., Bull. Am. Phys. Soc. 38 (1993) 2057.

[10] RAMAN, R., Canadian Fusion Fuels Technology Project, Mississauga, personal communication, 1997.

(Manuscript received 11 August 1997

Final manuscript accepted 30 January 1998)

E-mail address of J. Yee:

jyee@cco.caltechn.edu

Subject classification: J2, Tm; J2, Sm 